\title{
PULSED ELECTRICAL DISCHARGE PROPAGATION ALONG THE SURFACE OF LIQUID DIELECTRICTS
}

\author{
S. Gershman ${ }^{\mathrm{a}, \mathrm{b}}$, A Zwicker ${ }^{\mathrm{b}}$, and A. Belkind ${ }^{\mathrm{c}}$ \\ ${ }^{a}$ ARISE Inc., Scotch Plains, NJ 07076 U.S.A. \\ ${ }^{\mathrm{b}}$ Princeton Plasma Physics Laboratory, 100 Stellarator Road, Princeton, NJ 08540, U.S.A. \\ ${ }^{\mathrm{c}}$ A. Belkind and Associates, LLC, 184 Martins Way, North Plainfield, NJ 07060, USA
}

\begin{abstract}
Chemical and physical processes accompanying the propagation of electrical discharges along liquid surfaces are important for wide range of applications from electrical switching to liquid disinfection. $1-\mu \mathrm{s}, 10-15$ $\mathrm{kV}, 800 \mathrm{~ns}$ flat-top pulses are applied in a pin-to-plane electrode geometry. The discharge above and along a water surface is investigated in an Ar/Air mixture. The distilled water with conductivity of $5 \mu \mathrm{S} / \mathrm{cm}$ serves as a dielectric surface on the time scales of this experiment. The path of the streamer discharge from the needle to the flat (disk) electrode depends on the proximity of the water surface. The results of ICCD imaging with gate widths of $5-10$ ns show the discharge path transition from a straight horizontal path directly from the needle to plane to a curved path along the water surface. A $10 \mathrm{~nm}$ width bandpass filter selecting an Ar+ emission region is used to image the propagation of the ionization front and the build-up of plasma density. The time of the increase in plasma density corresponds to a sharp increase in the discharge current. The imaging results show distinct differences in the discharge development in the positive and negative polarity of the needle electrode. The novel aspect of this study is in combining a variety of timeresolved methods to study the dynamics of the streamer propagation along the surface of a liquid dielectric.
\end{abstract}

\section{I.INTRODUCTION}

Electrical discharges along liquid surfaces are important for wide range of applications from electrical switching to liquid disinfection [1, 2]. Examples include surface discharges on water surfaces and in gas bubbles for the treatment of polluted water [3-5]. Interaction of a discharge with a neighboring dielectric surface is seen also in an air discharge in the pin-to-surface electrode and other configurations [6].

Modeling and experiments have shown that pulsed discharge streamer propagation in gas bubbles liquids is affected by the relative dielectric permittivity of the liquid and the gas composition of the bubble [1, 2, 7-10].

Our previous experiments have been devoted to electrical discharge in gas bubbles in water [7-9]. The discharge in $\mathrm{Ar}, \mathrm{O}_{2}$, and air bubbles is generated by applying $1 \mu$ s long, $8-20 \mathrm{kV}$ rectangular voltage pulses to the needle electrode. Imaging, electrical and optical data show that the discharge is initiated in the needle region and propagates by the streamer mechanism through the bubble to the water (Fig. 1). Water acts as a dielectric barrier on the time scale of this experiment supporting the charge accumulation on the surface of the bubble. The length of the voltage pulse allows the discharge to extinguish itself, first by choking the electric field in the channel and then by dissipation of the energy of charged particles in the channel through collisions. A reverse discharge removes the accumulated charge from the water/gas interface when the applied voltage is turned off. The results support the modeling results from Babaeva, et al. [10].
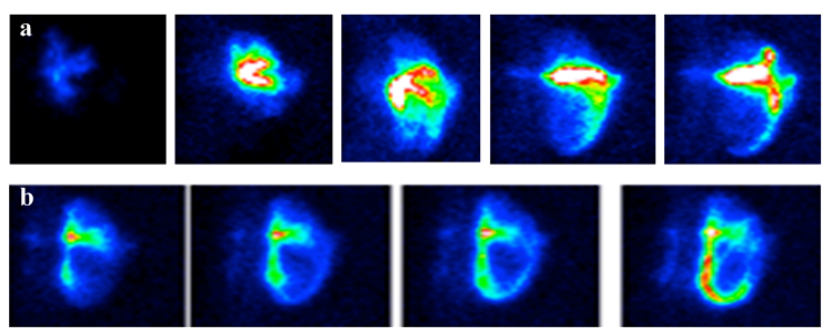

Figure 1. Consecutive images of discharge propagation in $\operatorname{Ar}$ (a: $5 \mathrm{~ns}$ exposure, $5 \mathrm{~ns}$ step) and $\mathrm{O}_{2}(\mathbf{b}: 2 \mathrm{~ns}$ exposure, 2 ns step) bubbles.

This paper presents results of an investigation of an electrical discharge in the needle-to-plane configuration in Ar/Air mixture in the presence of a water surface. Electrical characteristics and imaging results are described for positive and negative polarities of a needle electrode.

\section{EXPERIMENTAL SET UP}

The experimental setup consists of a electrically insulating chamber that has electrodes in a pin-to-plate horizontal configuration and is partially filled with water (Fig. 2). The setup allows one to change the pin to plate distance and the distance of the water level to the pin-toplate horizontal axis (Fig. 3). A stainless steel needle and a disk are used as electrodes. Although the discharge area is open to the air, an Ar flow can be introduced into the discharge through the needle. 


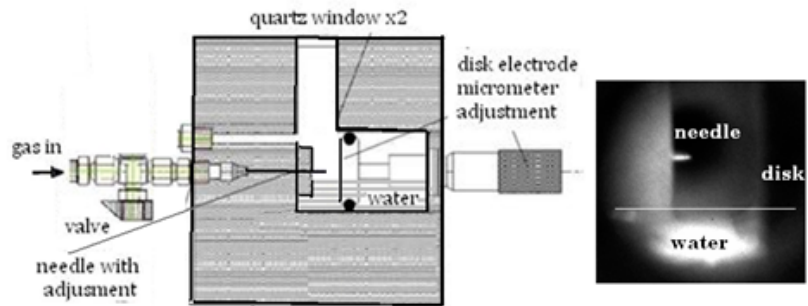

Figure 2. Schematic diagram of the experimental chamber for the study of the electrical discharges in a pin-to-plate horizontal geometry with various distances of water level to the pin-to-plate axis.

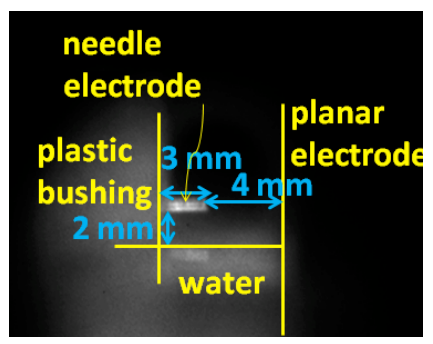

Figure 3. Electrode and water geometry. Measurements were taken by standard image analysis.

The electrical power delivery system consists of a dc high voltage power supply and a pulse shaping network (Fig. 4). The system creates positive or negative 10-15 $\mathrm{kV}$ and $1 \mathrm{~ms}$ long voltage pulses applied to the needle electrode (Fig. 5). The voltage across the load is measured by a Tektronix P6015A $75 \mathrm{MHz}$ HV probes; a Pearson high band pass current probe was used to measure current. A Tektronix TDS 3034 digital oscilloscope (300 $\mathrm{MHz}$ band pass and $2.5 \mathrm{GS} / \mathrm{s}$ sampling rate) is used to record current and voltage signals.

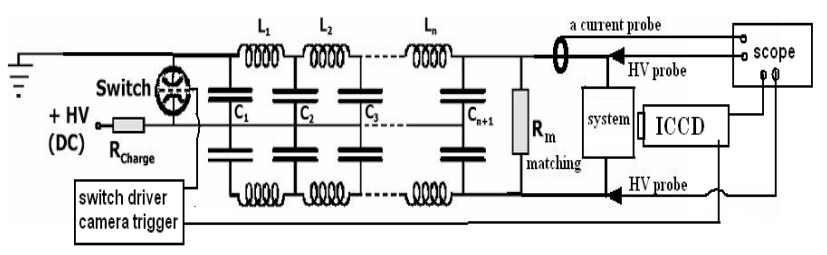

Figure 4. A schematic of the experimental setup.

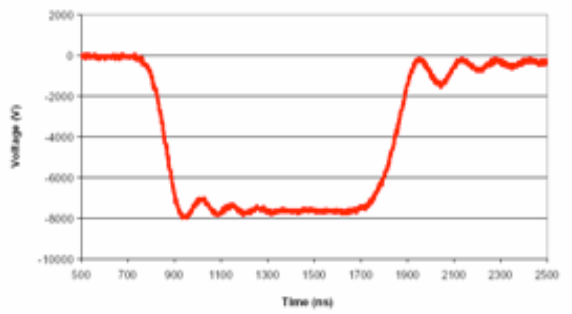

Figure 5. Voltage pulse applied between the needle and disk needle electrodes.
Imaging is conducted with a gated ICCD capable of taking images with exposure times down into the ns range. A sequential gating mode was used to study the time evolution of the discharge. The ICCD camera is triggered from the pulse driver with variable delay times.

The details of the experimental apparatus and techniques have been described previously [7-9].

\section{III.RESULTS AND DISCUSSION}

\section{A. Negative needle electrode}

In this set of experiments, the voltage was applied between the needle and the disk electrode with the needle at negative polarity. As seen in Fig. 5, the applied $1 \mathrm{~ms}$ voltage pulse has a ramp up and ramp down rates of about $100 \mathrm{~V} / \mathrm{ns}$. In the negative needle polarity, the discharge begins when the voltage reaches $-6400-/+200 \mathrm{~V}$, and the current rises at a rate of about $9 \times 10^{9} \mathrm{~A} / \mathrm{s}$ for the first $50 \mathrm{~ns}$ reaching a maximum value of about $600 \mathrm{~A}$ after $200 \mathrm{~ns}$.
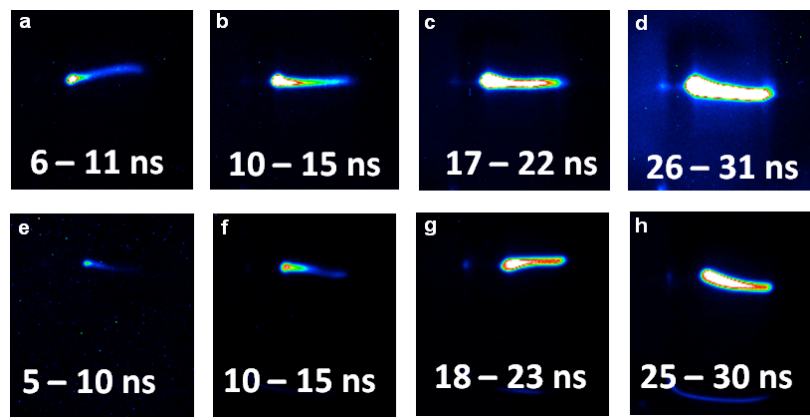

Figure 6. Top row $(\mathrm{a}-\mathrm{d})$ : ICCD $5 \mathrm{~ns}$ exposure (gate width) images of the discharge taken at different times during the discharge (as indicated). Bottom row $(e-h)$ : Images taken with an Ar+ bandpass filter. The distance between the needle electrode and water surface is $>4 \mathrm{~mm}$.

Images taken within the first few nanoseconds of the start of the current rise show that the discharge closes the 4 $\mathrm{mm}$ gap between the needle and the disk electrodes at a speed of about $(5+/-1) \times 10^{5} \mathrm{~m} / \mathrm{s}$. Examples of the time resolved images are shown in Fig. 6, a - d. In addition, images were taken with a bandpass filter selecting the $423+/-5 \mathrm{~nm}$ range corresponding primarily to $\mathrm{Ar}+$ emission. The images corresponding to Ar+ emission (Fig. 6, e-h) show an increase in intensity at the negative needle electrode extending with time to the positive disk electrode. The imaging results suggest fast initial streamer followed by an increase in ionization density (ion/electron concentration) propagating from the negative needle electrode to the positive disk. The images also suggest a possible formation of a cathode spot on the needle. The increase in the brightness and the width of the discharge channel corresponds to the time of rapid increase in the discharge current.

The water does not affect the discharge path if the needle-to-water distances are significantly greater than the 
inter-electrode distance. For the needle-to-disk distance of $4 \mathrm{~mm}$, a water surface lower than $4 \mathrm{~mm}$ below the needle, does not affect the path of the discharge. As the distance between the water surface and the needle electrode decreases, the discharge path bends toward the water surface. When the distance between the needle and water surface is about $2 \mathrm{~mm}$, the discharge propagates from the needle along the water surface to the disk anode (Fig. 7). The increase in brightness corresponding to a build-up of charge density also propagates from the cathode needle electrode to the disk cathode. The $10 \mathrm{~ns}$ exposure images show a bright spot forming on the disk anode.

The main features of the discharge with the negative needle include a fast initial streamer and an increase in ion/electron concentration that propagates from the negative needle electrode.
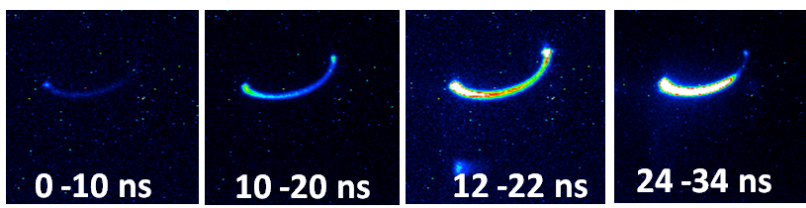

Figure 7. Sequential ICCD $10 \mathrm{~ns}$ gate width images of the discharge for the negative needle polarity. The water surface is approximately $2 \mathrm{~mm}$ below the needle.

\section{B. Positive needle electrode}

The entire experiment was repeated with the positive high voltage applied to the needle electrode. The starting voltage is about $5200+/-100 \mathrm{~V}$, lower than with the negative needle. The average rate of current rise for the initial $50 \mathrm{~ns}$ was about $7 \times 10^{9} \mathrm{~A} / \mathrm{s}$. The current reached a maximum of about 400 A after about 300 ns.

Similarly to the negative needle polarity, the discharge path is straight through the gas from the needle to the disk electrode when the water surface is far $(>4 \mathrm{~mm})$ below the needle electrode. The fast initial streamer remains evident in the images taken within the first several nanoseconds after the current starts to rise. Images taken sequentially over the following $40 \mathrm{~ns}$ show the discharge development progressing from both electrodes simultaneously (Fig. 8, a - f). Both the needle anode and the disk cathode develop bright spots. The bright regions grow toward the center of the discharge channel. The center of the channel brightens last. The images taken with Ar+ bandpass filter, show that the bright areas correspond to the regions with high plasma density.

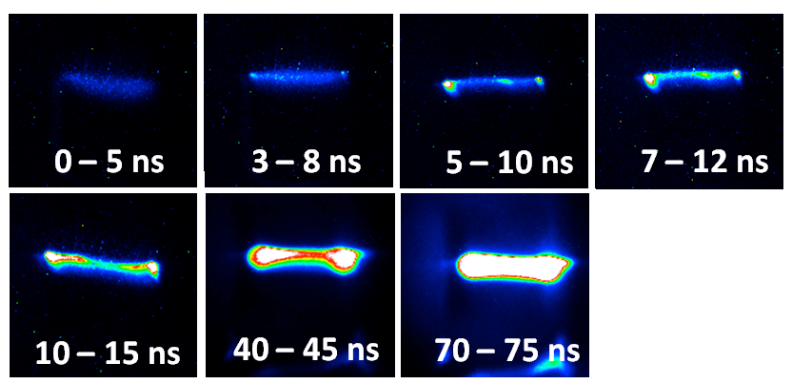

Figure 8. Sequential ICCD 5 ns gate width images of the discharge. The needle to water surface distance is $5 \mathrm{~mm}$.

Similarly to the negative needle polarity, the discharge channel curves toward the water when the water level is closer to the needle. At the needle-to-water surface distance of about $2 \mathrm{~mm}$, the discharge propagates along the surface of the water (Fig. 9, a - d). In case of a negative needle electrode, the discharge path always originated at the needle. In the case of a positive needle electrode, the discharge originates at the negative disk electrode and propagates along the surface. The discharge does not always "connect" to the needle electrode, terminating instead at the plastic wall of the experimental chamber. Another interesting feature of the discharge with the needle in positive polarity is the apparent possibility of several discharge paths occurring simultaneously. In these situations, the needle electrode glows brightly if the discharge propagates through the gas to the needle electrode (Fig. 9, e - g).
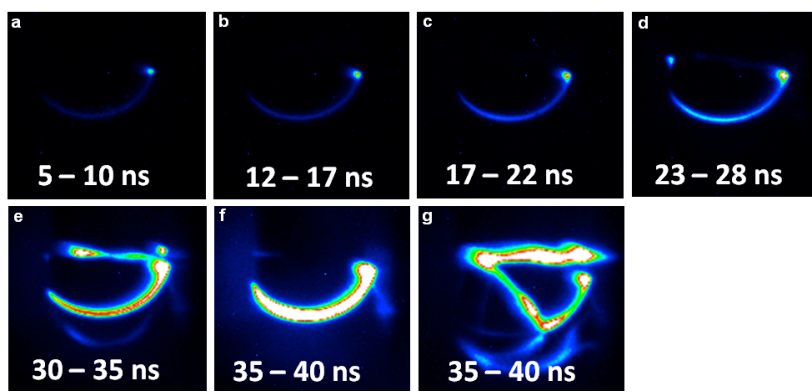

Figure 9. Sequential ICCD $5 \mathrm{~ns}$ gate width images of the discharge. The needle to water surface distance is $2 \mathrm{~mm}$.

The main new features revealed by the imaging of the discharge with the needle as the anode include the following: 1) the density increase propagates from both electrodes toward the middle of the discharge channel when the discharge propagates through the gas directly; 2) the discharge along the water surface increases in brightness from the negative disk electrode and often terminates at the chamber wall, not at the needle; 3 ) several simultaneous paths are apparent in the same discharge. 


\section{IV.SUMMARY}

In this paper we present preliminary results of an investigation into the propagation of an electrical discharge in the pin to plane geometry in close proximity of the water surface. The $5 \mathrm{~ns}$ and $10 \mathrm{~ns}$ exposure times show that the discharge propagates directly through the air when the distance to the water surface is equal to or greater than the distance from the needle to the disk. The discharge path bends toward the water surface as the distance between the needle and the surface decreases and at distances of about $2 \mathrm{~mm}$ in our geometry, the discharge propagates along the water surface. This behavior does not depend on the polarity of the needle electrode. The discharges exhibit significant other differences for different polarities of the needle. When the cathode is at the needle electrode, the plasma density appears to increase from the needle to the disk. The plasma density propagates from the disk and the needle toward the center of the discharge channel for the needle anode.

The shape of the electric field in close proximity to the water surface changes dynamically due to the photoionization of the liquid surface, the deposition of fast moving electrons, surface properties of the liquid, and other factors $[11,12]$. These factors are therefore expected to affect the path of the discharge. The differences in the propagation of the discharge through the air in the two polarities may be influenced by field emission from the cathode. Additional experiments are needed to sort out these possible influences.

\section{REFERENCES}

[1] V. I. Parvulescu, M. Magureanu, P. Lukes, "Plasma Chemistry and Catalysis in Gases and Liquids" Ed 2, John Wiley \& Sons, 2012.

[2] Yong Yang; Young I. Cho, Alexander Fridman, Plasma Discharge in Liquid: Water Treatment and Applications, CRC Press, 2012.

[3] F. Tochikubo, Y. Furuta, S. Uchida and T. Watanabe, Study of Wastewater Treatment by $\mathrm{OH}$ Radicals Using DC and Pulsed Corona Discharge over Water, Jpn. J. Appl. Phys. Vol. 45 (no. 4A), pp. 2743-2748, 2006.

[4] M. Sato, Environmental and biotechnological applications of high-voltage pulsed discharges in water, Plasma Sources Sci. Technol.,17, 024021 (7 pp), 2008.

[5] O. Mozgina, A. Koutsospyros, S. Gershman, A. Belkind, C. Christodoulatos, and K. Becker, IEEE Trans. Plasma Sci., 37 (no. 6) pp. 905-910, 2009.

[6] V. Bloshchitsyn, Review of surface discharge experiments, St.-Petersburg State University, Physics Faculty, 2010 (http://arxiv.org/pdf/1005.5044.pdf).

[7] S. Gershman, O. Mozgina, A. Belkind, K. Becker, and E. Kunhardt, Electrical Discharges in Bubbled
Water., Contrib. Plasma Phys., 46 (no. 1-2), pp. 1-7, 2007.

[8] S. Gershman, A. Belkind, and K. Becker, Optical emission diagnostics of the plasma channel in a pulsed electrical discharge in a gas bubble, IEEE Trans. Pulsed Power, Pulsed Power Conference (Washington, DC), pp. 838-843, 2009.

[9] S. Gershman and A. Belkind, Time-resolved processes in a pulsed electrical discharge in argon bubbles in water, Eur. Phys. J., D 60, pp. 661-672, 2010.

[10] N. Babaeva and M.J. Kushner, Structure of positive streamers inside gaseous bubbles immersed in liquids, J. Phys. D: Appl. Phys., 42, 132003 (5pp), 2009.

[11] V. M. Shmelev and A. D. Margolin, agation of an Electric Discharge over the Surface of Water and Semiconductor, High Temperature, 41 (6), pp. 735-741, 2003.

[12] V. Bloshchitsyn, Review of surface discharge experiments, St.-Petersburg State Univ., Physics Faculty, 2010, http://arxiv.org/pdf/1005.5044.pdf. 